\title{
$X$ Chromosome-Inactivation Patterns in 31 Individuals with PHACE Syndrome
}

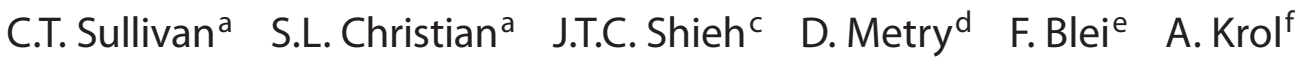 \\ B.A. Drolet ${ }^{g}$ I.J. Frieden $^{b}$ W.B. Dobyns ${ }^{a, b}$ D.H. Siegel ${ }^{f}$
}

${ }^{a}$ Center for Integrative Brain Research, Seattle Children's Research Institute, and b Departments of Pediatrics and Neurology, University of Washington, and Center for Integrative Brain Research, Seattle Children's Research Institute, Seattle, Wash., 'Division of Medical Genetics, Department of Pediatrics and Institute for Human Genetics, University of California San Francisco, San Francisco, Calif., dDepartment of Dermatology and Pediatrics, Texas Children's Hospital, Houston, Tex., 'Vascular Birthmark Institute of New York, St. Luke's Roosevelt Hospital, New York, N.Y., ' Department of Dermatology, Oregon Health and Science University, Portland, Oreg., ${ }^{9}$ Department of Dermatology and Pediatrics, Medical College of Wisconsin, Children's Hospital of Wisconsin, Milwaukee, Wisc., USA

\section{Key Words}

Coarctation of the aorta $\cdot$ Hemangioma $\cdot$ PHACE

syndrome $\cdot$ Posterior fossa anomaly $\cdot X$-inactivation

\begin{abstract}
Segmental hemangiomas of the head and neck can be associated with multiple congenital anomalies in the disorder known as PHACE syndrome (OMIM 606519) (posterior fossa malformations, hemangioma, arterial anomalies, cardiac defects, and eye anomalies). All reported cases of PHACE syndrome to date have been sporadic, and the genetic basis of this disorder has not yet been established. PHACE syndrome has a striking female predominance which has raised the question of X-linked inheritance. In this study, the X chromosome-inactivation $(\mathrm{XCl})$ patterns of 31 females with PHACE syndrome and their mothers were analyzed using blood-derived DNA and X-chromosome locus methylation assay. This study was performed to test the hypothesis that some cases of PHACE syndrome are due to X-linked inheritance and favorable skewing in the mothers may protect against a severe
\end{abstract}

phenotype, but the clinical phenotype may be unmasked in daughters with a random pattern of $\mathrm{X}$-inactivation. $\mathrm{XCl}$ analysis was informative in $27 / 31$ mothers. Our results identified skewed XCl in 5 of 27 (19\%) informative mothers, which is not statistically significant with a $p$ value of 0.41 . None of the mothers reported significant medical problems, although a full PHACE work-up has not been performed in these individuals. Skewed $\mathrm{XCl}$ in the mothers of children with PHACE was identified in only a minority of cases. Based on these results, genetic heterogeneity is likely in PHACE syndrome, although it is possible a subset of cases are caused by a mutation in an X-linked gene.

Copyright $\odot 2012$ S. Karger AG, Basel

Segmental hemangiomas of the head and neck can be associated with malformations of the heart, cerebral arteries, brain, and eyes [Haggstrom et al., 2010]. This association was first described in a partial form consisting of facial hemangiomas and cranial arterial anomalies, expanded by adding Dandy-Walker malformation, and lat-

\section{KARGER}

E-Mail karger@karger.com

www.karger.com/msy
(C) 2012 S. Karger AG, Basel

1661-8769/13/0043-0114\$38.00/0
Dawn Siegel, MD

8701 Watertown Plank Road

TBRC 2nd Floor, C2010

Milwaukee, WI 53226 (USA)

E-Mail dsiegel@mcw.edu 
er designated as PHACE (posterior fossa anomalies, hemangioma, arterial anomalies, cardiac anomalies/coarctation of the aorta, eye anomalies, and sternal defects) syndrome [Frieden et al., 1996]. More than 300 patients with PHACE syndrome have been reported in the literature. In 2008, a multidisciplinary group of specialists convened to develop a consensus statement on the diagnostic criteria for PHACE syndrome [Metry et al., 2009]. A diagnosis of definite PHACE syndrome is based on a facial hemangioma larger than $5 \mathrm{~cm}$ plus 1 major or $2 \mathrm{mi}-$ nor criteria (table 1). Several case series and prospective studies have described skewing of the sex ratio toward females [Gorlin et al., 1994; Hemangioma Investigator Group et al., 2007], with an initial reported 8:1 female to male ratio [Metry et al., 2006]. More recently, larger studies on individuals with PHACE, as well as data from the PHACE Syndrome Clinical Registry and DNA Repository with 150 subjects enrolled, have reported a slightly lower female to male ratio at 5.6:1 [Haggstrom et al., 2010].

Levin et al. [2007] reported a case of a female infant with a diagnosis of PHACE syndrome based on perioral hemangiomas as well as hemangiomas on the chest and extremities, cerebrovascular anomalies, sternal scar and supraumbilical raphe. X chromosome-inactivation (XCI) studies showed a random XCI pattern in the affected child and a skewed pattern in the mother, suggesting that the skewed XCI in the mother may have been protective [Levin and Kaler, 2007]. Given the female predominance and the previous case report of skewed XCI in PHACE syndrome, the question of whether XCI could have a broader role in the pathogenesis of PHACE has been raised [Levin and Kaler, 2007]. In general, $\mathrm{XCI}$ is expected to be random. It occurs during the blastocyst stage in female development when one of $2 \mathrm{X}$ chromosomes in each cell is silenced [Hendrich et al. 1997; Panning et al., 1997]. However, when a mutation is present on one $\mathrm{X}$ chromosome, that cell population may have a growth disadvantage, resulting in skewed XCI. Thus, in some cases, nonrandom skewing away from a disease allele may be protective. The Xlinked neurodevelopmental condition, Rett syndrome, has been used as a model to study the influence of XCI [Young and Zoghbi, 2004]. In some reports, skewed XCI has been associated with a milder Rett phenotype, suggesting that suppression of the mutated allele was protective [Ishii et al., 2001]. In addition, in certain classes of X-linked mental retardation disorders, the carriers have a high rate of skewed (>80:20) XCI with skewing against the cells with the mutation [Plenge et al., 2002].
In this study, we performed XCI studies in 31 motherdaughter duos to evaluate the patterns of X-inactivation in PHACE syndrome.

\section{Methods}

Subjects

This study was approved by the Children's Hospital of Wisconsin Institutional Review Board and IRBs of collaborating institutions. Subjects were all enrolled in the Genetic Analysis of PHACE Syndrome study as part of the PHACE Syndrome International Clinical Registry and DNA Repository. Subjects were selected for this X-inactivation study from the PHACE syndrome International DNA Repository. All met published diagnostic criteria outlined in table 1.

\section{DNA Extraction}

Peripheral blood was obtained at the time of enrollment in the Genetic Analysis of PHACE Syndrome study. The Puregene DNA Purification kit was used to isolate DNA using the manufacturer's protocol.

\section{$X$-Inactivation Studies}

The $2 \mathrm{X}$ chromosomes in human females can be distinguished from each other by using a polymorphic CAG repeat on exon 1 of AR (human androgen receptor) in Xq12. The XCI status of each allele is measured by comparing their relative levels of $\mathrm{CpG}$ methylation, using the methylation-sensitive restriction enzyme HpaII. Genomic DNA was digested with either RsaI alone or RsaI with HpaII, and digests were PCR amplified as previously described [Amos-Landgraf et al., 2006]. RsaI is used as a background enzyme; it digests genomic DNA, but does not affect fragment size, as the PCR amplified region does not contain an RsaI cutting site. All PCRs were done in duplicate. The forward primer was labeled with FAM on the $5^{\prime}$ end. PCR products were analyzed on an ABI 3730 XL DNA sequencing analyzer at Seattle BioMed Research Institute and processed using GeneMarker 1.9 software (SoftGenetics). The X-inactivation level of each allele was calculated using peak heights with the formulas $\mathrm{X}_{1}=[\mathrm{d} 1 / \mathrm{u} 1] /[(\mathrm{d} 1 / \mathrm{u} 1)+(\mathrm{d} 2 / \mathrm{u} 2)]$ and $\mathrm{X}_{2}=[\mathrm{d} 2 / \mathrm{u} 2] /[(\mathrm{d} 1 /$ $\mathrm{u} 1)+(\mathrm{d} 2 / \mathrm{u} 2)]$, where $\mathrm{d}$ and $\mathrm{u}$ denote HpaII-digested and undigested DNA respectively (fig. 1). The X-inactivation ratios $\left(\mathrm{X}_{1}: \mathrm{X}_{2}\right)$ represent the level of inactivation of the smaller allele compared to that of the larger allele. Following standard practice, random XCI was defined as 50:50 to 79:31, skewed XCI as 80:20 or larger, and highly skewed as 90:10 or larger. Nine individuals had alleles with only one CAG-repeat different in size (fig. 1), which makes exact ratios difficult to quantify due to overlapping and lower molecular weight (MW) stutter-peak artifacts present in short tandem repeat PCRs [Amos-Landgraf et al., 2006]. The stutter peak heights in our experiments were generally $33 \%$ or less of the peak height of the true allele. To correct for stutter peaks in these 4 cases only, 33\% of the height of the larger MW allele was subtracted from the smaller MW allele before calculating the XI ratio: $\mathrm{u}_{\text {corrected }}=\mathrm{u} 1-0.33 \times \mathrm{u} 2$, and $\mathrm{d} 1_{\text {corrected }}=\mathrm{d} 1-0.33 \times \mathrm{d} 2$. 
Fig. 1. XI Assay: Microsatellite PCR products of AR are shown with (right) and without (left) HpaII digestion. Arrows indicate peaks u1 (red), u2 (blue), d1 (orange), and $\mathrm{d} 2$ (green) as described in Methods. Individual 08-251.0 had the most skewed XI ratio in this study, at 07:93.

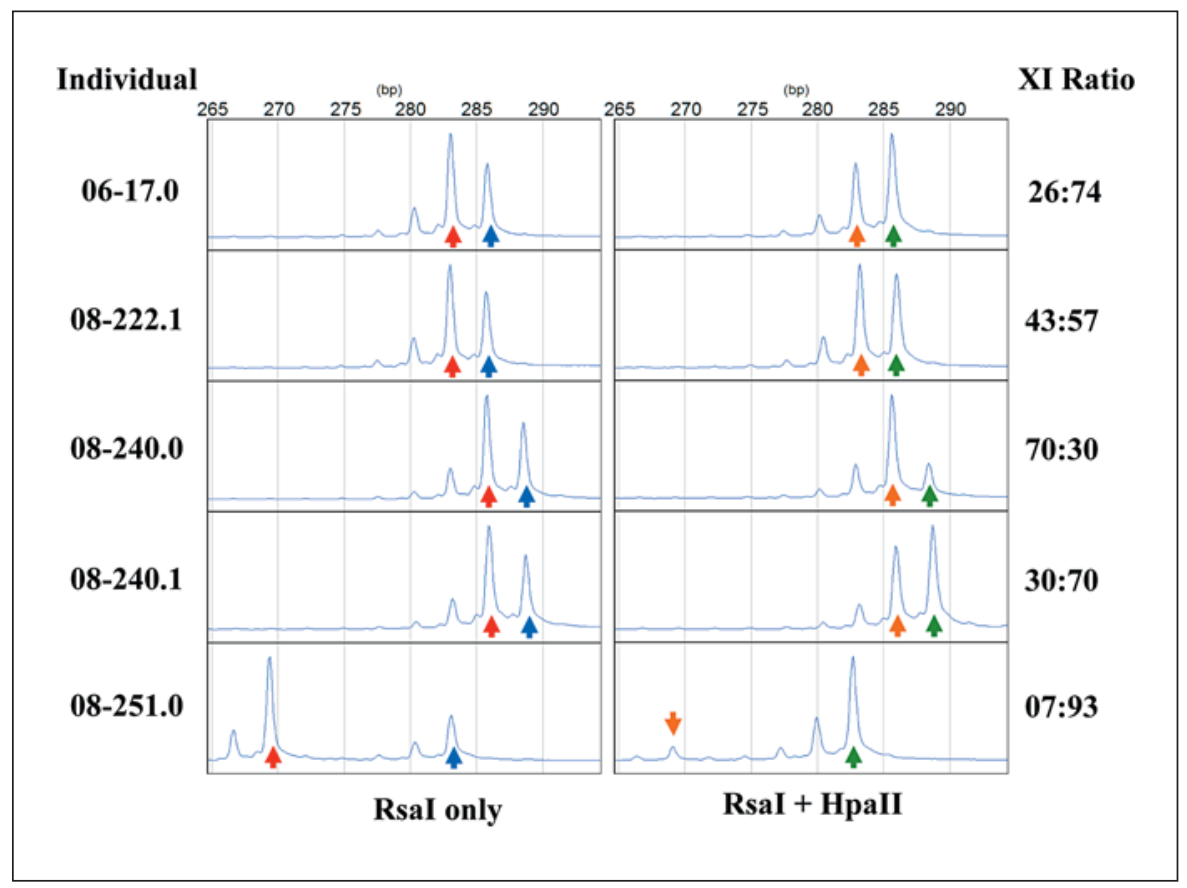

Table 1. Major and minor diagnostic criteria of PHACE syndrome

\begin{tabular}{lr}
\hline Criteria & $\%$ \\
\hline Major & \\
Segmental hemangioma of the head & 100 \\
Dysplasia of large cerebral artery & 14 \\
Stenosis, occlusion, absence or hypoplasia of a large & \\
$\quad$ cerebral artery & 60 \\
Posterior fossa anomaly & 35 \\
Aortic arch anomaly & 28 \\
Anomalous subclavian artery & 14 \\
Posterior segment anomalies of the eye & 8 \\
Sternal defect & 22 \\
Umbilical raphe & 9 \\
Minor & \\
Persistent embryonic arteries other than trigeminal & 7 \\
Midline anomalies & 2 \\
Disorder of migration & 6 \\
Ventricular septal defect & 13 \\
Anterior segment defect of the eye & 8 \\
Sternal papule & 1.5
\end{tabular}

The Hemangioma Investigator Group published a consensus statement on the diagnostic criteria of PHACE syndrome [Metry et al., 2009]. Definite PHACE = Segmental hemangioma of the head $>5 \mathrm{~cm}$ plus 1 major or 2 minor criteria; possible PHACE $=$ segmental hemangioma of the head $>5 \mathrm{~cm}$ plus 1 minor criteria [modified with permission].

\section{Results}

\section{Demographic Information}

Our cohort consisted of 31 females with PHACE syndrome and their mothers. The reported ethnicities for the probands were as follows: Caucasian non-Hispanic (25), Caucasian-Hispanic (4), African-American (1), and Asian (1). The age range of the mothers at the time of pregnancy was 21-39 years.

\section{Phenotypic Characteristics of the Cohort}

Based on inclusion criteria, all subjects had a segmental hemangioma on the head and neck. Structural brain anomalies were reported in 14/31 (45\%). Imaging of the cerebral arteries was available for $29 / 31$ probands. Cerebrovascular anomalies were reported in 25/27 (92\%). A total of 11/31 (35\%) subjects reported congenital heart defects and 4 reported aberrant subclavian arteries. Cardiac and aortic anomalies included: coarctation (5), dilated aorta (2), tetralogy of Fallot (1), VSD (1), and right aortic arch (2). Eye anomalies were reported in 14/31 (45\%). Sternal defects were only reported in 4/31 (13\%) subjects.

\section{X-Inactivation Results}

$\mathrm{XCI}$ analysis was informative in 27/31 mothers; 4 mothers were homozygous and, thus, uninformative by our assay. Primary data are shown in figure 1 and the results are
Sullivan/Christian/Shieh/Metry/Blei/ Krol/Drolet/Frieden/Dobyns/Siegel 
Table 2. X-chromosome inactivation results in families of children with PHACE syndrome

\begin{tabular}{|c|c|c|c|c|}
\hline Proband & Sex & $\mathrm{XCI}$ ratio & Mother & $\mathrm{XCI}$ ratio \\
\hline $06-17.0$ & $\mathrm{~F}$ & $26: 74$ & 06-17.1 & $31: 69$ \\
\hline $06-11.0$ & $\mathrm{~F}$ & $62: 38$ & $06-11.1$ & $16: 84$ \\
\hline $08-205.0$ & $\mathrm{~F}$ & $62: 38$ & $08-205.1$ & $50: 50$ \\
\hline $08-211.0$ & $\mathrm{~F}$ & $68: 32$ & $08-211.1$ & $20: 80$ \\
\hline $08-222.0$ & $\mathrm{~F}$ & $61: 39$ & $08-222.1$ & $43: 57$ \\
\hline $08-240.0$ & $\mathrm{~F}$ & $70: 30$ & $08-240.1$ & $30: 70$ \\
\hline $08-249.0$ & $\mathrm{~F}$ & $63: 37$ & $08-249.1$ & $74: 26$ \\
\hline 08-301.0 & $\mathrm{F}$ & $66: 34$ & $08-301.1$ & $40: 60$ \\
\hline 08-305.0 & $\mathrm{F}$ & $83: 17$ & 08-305.1 & $23: 77$ \\
\hline $08-307.0$ & $\mathrm{~F}$ & $48: 52$ & 08-307.1 & $33: 67$ \\
\hline 08-308.0 & $\mathrm{F}$ & $32: 68$ & $08-308.1$ & $63: 37$ \\
\hline 08-309.0 & $\mathrm{F}$ & $51: 49$ & 08-309.1 & $88: 12$ \\
\hline 08-310.0 & $\mathrm{F}$ & $45: 55$ & $08-310.1$ & $45: 55$ \\
\hline $08-318.0$ & $\mathrm{~F}$ & $47: 53$ & $08-318.1$ & $61: 39$ \\
\hline $08-251.0$ & $\mathrm{~F}$ & 07:93 & $08-251.1$ & $13: 87$ \\
\hline 08-303.0 & $\mathrm{F}$ & $52: 48$ & 08-303.1 & $66: 34$ \\
\hline $08-062.0$ & $\mathrm{~F}$ & $57: 43$ & $08-312.1$ & $68: 32$ \\
\hline $08-241.0$ & $\mathrm{~F}$ & $70: 30$ & $08-241.1$ & $85: 15$ \\
\hline 08-202.0 & $\mathrm{F}$ & $52: 48$ & $08-202.1$ & UI \\
\hline $08-028.0$ & $\mathrm{~F}$ & $67: 33$ & $08-028.1$ & $36: 64$ \\
\hline LR03-223 & $\mathrm{F}$ & $64: 36$ & LR03-223m & $43: 57$ \\
\hline LR03-294 & $\mathrm{F}$ & $61: 39$ & LR03-294m & $73: 27$ \\
\hline LR04-119 & $\mathrm{F}$ & $63: 37$ & LR04-119m & UI \\
\hline LR05-117 & $\mathrm{F}$ & ND & LR05-117m & $25: 75$ \\
\hline B061.0 & $\mathrm{F}$ & $84: 16$ & B061.1 & $71: 29$ \\
\hline В084.0 & $\mathrm{F}$ & UI & B084.1 & $51: 49$ \\
\hline B138.0 & $\mathrm{F}$ & $62: 38$ & B138.1 & UI \\
\hline 08-302.0 & $\mathrm{F}$ & $59: 41$ & 08-302.1 & $50: 50$ \\
\hline $08-325.0$ & $\mathrm{~F}$ & $50: 50$ & $08-325.1$ & UI \\
\hline B512.0 & $\mathrm{F}$ & $64: 36$ & B512.1 & $66: 34$ \\
\hline B703.0 & $\mathrm{F}$ & $34: 66$ & B703.1 & $55: 45$ \\
\hline
\end{tabular}

Cases with XCI skewing of 80:20 or larger are bolded. UI = Uninformative due to homozygosity at AR locus; $\mathrm{ND}=$ testing was not done.

summarized in table 2 . We found skewed XCI in lymphocytes in 5 of the 27 (19\%) informative mothers, which is slightly more than the expected population average of $15 \%$ determined in an analysis of 415 healthy adult female controls, although not statistically significant with a p value of 0.41 (Fisher's exact test) [Amos-Landgraf et al., 2006]. XCI studies were informative in 29/31 female probands for which we had DNA. We found skewed XCI in 3 of 29 (10\%), which is not statistically significantly different than the expected population average of $5 \%$ determined from analysis of 590 healthy newborn girls with a value of 0.23 (Fisher's exact test) [Amos-Landgraf et al., 2006]. The remaining mothers and probands had random patterns of XCI.

\section{Discussion}

PHACE syndrome has emerged as an important neurocutaneous and neurovascular syndrome, occurring in approximately $30 \%$ of infants with segmental hemangiomas of the head and neck [Haggstrom et al., 2010]. The incidence of PHACE syndrome has likely been underestimated. In a prospective study performed in pediatric dermatology clinics, ascertainment of PHACE was higher than that of Sturge-Weber syndrome, suggesting that PHACE is more common than latter [Metry et al., 2006]. The pathogenesis of PHACE syndrome is unknown; however, risk factors include female sex (female:male ratio $=5.6: 1)$, white non-Hispanic ethnicity $(68 \%)$ and premature birth (16\%) [Metry et al., 2008]. Although there is a female predominance, the reported severity of affected males seems comparable to affected females. One possible exception is the slightly higher occurrence of brain malformations in males with PHACE [Metry et al., 2008]. No specific environmental influences have been identified, and to date, all reports of PHACE syndrome have been sporadic. One prior case report demonstrating skewed X-inactivation in the mother of a female with PHACE syndrome raised the question of whether skewed $\mathrm{XCI}$ may play a protective role in the mothers of children with PHACE [Levin and Kaler, 2007]. In this study, we follow-up on that report by performing XCI studies on a group of 31 females with PHACE syndrome and their mothers. Although there was a slight increase in the number of mothers and daughters with skewed XCI compared with the expected population average, these findings were not statistically significant. Despite the female predominance in PHACE syndrome, we did not find a significant increase in skewed XCI in the probands or their mothers. In considering the pathogenetic mechanism in PHACE syndrome, several observations may provide relevant clues. The most common and consistent abnormalities involve arteriopathy of the great vessels, with the most severely affected patients having extensive bilateral involvement of brain, neck, chest, gastrointestinal tract, and mesenteric arteries. Less severely affected patients may have unilateral localized involvement. The regional localization of findings in the majority of cases has led some to suggest a developmental field defect or postzygotic mosaicism as a possible mechanism in PHACE [Metry et al., 2006]. The striking regional variation observed in patients with $\mathrm{PHACE}$, and related hemangioma syndromes, lends considerable support to a mosaic hypothesis. Several other wellknown developmental disorders may be caused by either 
germline or mosaic mutations of the causal gene. For example, both germline and mosaic mutations have been observed in focal dermal hypoplasia, an X-linked syndrome caused by mutations in the PORCN gene [Grzeschik et al., 2007].

In summary, skewed XCI in the mothers of children with PHACE was identified in only a minority of cases; therefore, we conclude maternal skewed XCI does not highly correlate with most cases of PHACE syndrome. Further studies using next-generation sequencing may provide further insight into the underlying pathogenetic mechanism in PHACE syndrome.

\section{Acknowledgements}

We would like to thank the families who participated in this study. We are grateful to Gina Im and Shawna Joachim for their assistance with data management. D.H.S. was supported by the Oregon Clinical Translational Research Institute (grant UL1 RR024140 from the National Center for Research Resources (NCRR), a component of the National Institutes of Health (NIH), and NIH Roadmap for Medical Research), Dermatology Foundation Career Development Award and Patient Directed Investigator Award and the Society for Pediatric Dermatology William L. Weston Research Grant. This study was supported in part by a grant from the NIH to Dr. Dobyns (2R01-NS046616). J.T.C.S. is supported by NIH NHLBI.

\section{References}

Amos-Landgraf JM, Cottle A, Plenge RM, Friez $\mathrm{M}$, Schwartz CE, et al: X chromosome-inactivation patterns of 1,005 phenotypically unaffected females. Am J Hum Genet 79:493499 (2006).

Frieden IJ, Reese V, Cohen D: PHACE syndrome. The association of posterior fossa brain malformations, hemangiomas, arterial anomalies, coarctation of the aorta and cardiac defects, and eye abnormalities. Arch Dermatol 132:307-311 (1996).

-Gorlin RJ, Kantaputra P, Aughton DJ, Mulliken JB: Marked female predilection in some syndromes associated with facial hemangiomas. Am J Med Genet 52:130-135 (1994).

-Grzeschik KH, Bornholdt D, Oeffner F, König A, del Carmen Boente M, et al: Deficiency of PORCN, a regulator of Wnt signaling, is associated with focal dermal hypoplasia. Nat Genet 39:833-835 (2007).

Haggstrom AN, Garzon MC, Baselga E, Chamlin SL, Frieden IJ, et al: Risk for PHACE syndrome in infants with large facial hemangiomas. Pediatrics 126:e418-426 (2010).
Hemangioma Investigator Group, Haggstrom AN, Drolet BA, Baselga E, Chamlin SL, et al: Prospective study of infantile hemangiomas: demographic, prenatal, and perinatal characteristics. J Pediatr 150:291-294 (2007).

Hendrich BD, Plenge RM, Willard HF: Identification and characterization of the human XIST gene promoter: implications for models of X chromosome inactivation. Nucleic Acids Res 25:2661-2671 (1997).

Ishii T, Makita Y, Ogawa A, Amamiya S, Yamamoto $\mathrm{M}$, et al: The role of different $\mathrm{X}$-inactivation pattern on the variable clinical phenotype with Rett syndrome. Brain Dev 23 Suppl 1:S161-164 (2001).

Levin JH, Kaler SG: Non-random maternal Xchromosome inactivation associated with PHACES. Clin Genet 72:345-350 (2007).

Metry DW, Haggstrom AN, Drolet BA, Baselga E, Chamlin S, et al: A prospective study of PHACE syndrome in infantile hemangiomas: demographic features, clinical findings, and complications. Am J Med Genet A 140:975-986 (2006).
- Metry DW, Siegel DH, Cordisco MR, Pope E, Prendiville J, et al: A comparison of disease severity among affected male versus female patients with PHACE syndrome. J Am Acad Dermatol 58:81-87 (2008).

Metry DW, Heyer G, Hess C, Garzon M, Haggstrom A, et al: Consensus Statement on Diagnostic Criteria for PHACE Syndrome. Pediatrics 124:1447-1456 (2009).

- Panning B, Dausman J, Jaenisch R: X chromosome inactivation is mediated by Xist RNA stabilization. Cell 90:907-916 (1997).

- Plenge RM, Stevenson RA, Lubs HA, Schwartz CE, Willard HF: Skewed X-chromosome inactivation is a common feature of X-linked mental retardation disorders. Am J Hum Genet 71:168-173 (2002).

-Young JI, Zoghbi HY: X-chromosome inactivation patterns are unbalanced and affect the phenotypic outcome in a mouse model of Rett syndrome. Am J Hum Genet 74:511-520 (2004) 